\title{
Reduced and projected two-particle entanglement at finite temperatures
}

\author{
P. Samuelsson ${ }^{1}$, I. Neder ${ }^{2}$, M. Büttiker ${ }^{3}$ \\ ${ }^{1}$ Division of Mathematical Physics, Lund University, Box 118, S-221 00 Lund, Sweden \\ ${ }^{2}$ Physics Department, Harvard University, Cambridge, Massachusetts 02138, USA and \\ ${ }^{3}$ Département de Physique Théorique, Université de Genève, CH-1211 Genève 4, Switzerland
}

\begin{abstract}
We present a theory for two-particle entanglement production and detection in mesoscopic conductors at finite temperature. The entanglement of the density matrix projected out of the emitted many-body state differs from the entanglement of the reduced density matrix, detectable by current correlation measurements. Under general conditions reduced entanglement constitutes a witness for projected entanglement. Applied to the recent experiment [Neder et al, Nature 448333 (2007)] on a fermionic Hanbury Brown Twiss two-particle interferometer we find that despite an appreciable entanglement production in the experiment, the detectable entanglement is close to zero.
\end{abstract}

PACS numbers: 73.23.-b, 05.40.-a, 72.70.+m, 74.40.+k

The last decade has witnessed an increasing interest in generation and detection of entanglement in mesoscopic conductors [1, 2]. Entanglement is an ubiquitous quantum effect, it describes correlations between particles that can not be accounted for classically. A better understanding of entanglement of elementary charge carriers, or quasiparticles, is therefore of fundamental interest. Due to controllable system properties and coherent transport conditions, mesoscopic conductors constitute ideal systems for the investigation of quasiparticle entanglement. In a longer time perspective, the prospect of quantum information processing using spin or orbital quantum states of individual quasiparticles provides additional motivation for such an investigation.

To date quasiparticle entanglement has remained experimentally elusive. However, recently an important step was taken towards a demonstration of entanglement in mesoscopic conductors. Based on the theoretical proposal [3] for a fermionic two-particle interferometer (2PI), see Fig. [1] Neder et al [4] were able to demonstrate interference between two electrons emitted from independent sources. In perfect agreement with theory, the interference pattern was visible in the current correlations but not in the average current. Under conditions of zero dephasing and temperature, the part of the emitted state with one electron in each detection region $\mathrm{A}, \mathrm{B}$ would be

$$
\left|\Psi_{s}\right\rangle=2^{-1 / 2}\left(|1\rangle_{A}|2\rangle_{B}-|2\rangle_{A}|1\rangle_{B}\right)
$$

where 1,2 denote the sources. The wavefunction $\left|\Psi_{s}\right\rangle$ is maximally entangled, it is a singlet in orbital, or pseudo spin, space. However, in the experiment [4] a reduced amplitude $(\sim 25 \%)$ of the current correlation oscillations was observed, suggesting an important effect of both dephasing and finite temperature. This raises two interesting and interrelated questions: are the electrons reaching the detectors at $\mathrm{A}$ and $\mathrm{B}$ entangled and if so, can this two-particle entanglement be unambiguously detected by measurements of currents and current correlators?

In this work we provide an affirmative answer to both these questions. We present a general theory for two- particle entanglement generation and detection in mesoscopic conductors at finite temperatures and apply it to the 2 PI. Under very general conditions a nonzero entanglement of the reduced orbital density matrix, accessible by current and current correlation measurements [5], is shown to be a signature of finite entanglement of the density matrix projected out from the emitted many-body state. In other words, finite reduced entanglement constitutes a witness for finite projected entanglement. For the 2PI-experiment, while the projected density matrix is found to be clearly entangled, the reduced, observable density matrix is only marginally entangled.
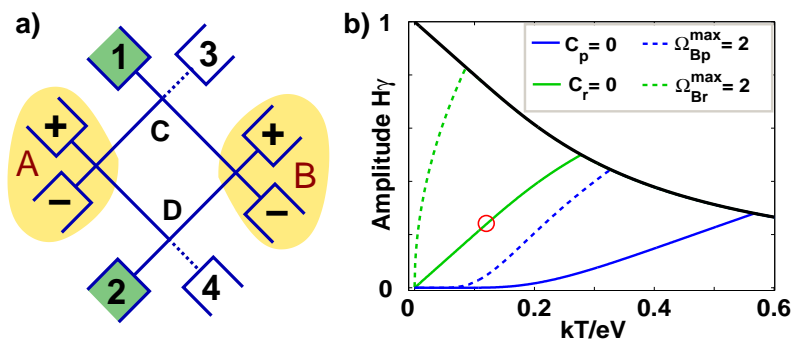

FIG. 1: (color online) a) Schematic of the two-particle interferometer (2PI) 3] with beam splitters C,D and biased (grounded) contacts 1,2 $(3,4)$. Detector regions A and B (yellow shaded) contain beam splitters and grounded contacts \pm . b) Amplitude-temperature plot for the 2PI with semi-transparent splitters C,D. Above/to the left of the plotted lines the entanglement of the projected (reduced) density matrix is finite, $C_{p}>0\left(C_{r}>0\right)$, and a Bell inequality is violated, $\Omega_{B p}^{\max }>2\left(\Omega_{B r}^{\max }>2\right)$. The red ring denotes the parameters of the experiment [4], showing that while the emitted state is clearly entangled, $C_{p}>0$, it is barely detectable by current and current correlation measurements, $C_{r} \approx 0$.

We are interested in finite temperature orbital [6] entanglement in a general mesoscopic system, shown in Fig. 3. A theory for entanglement production in noninteracting [7] mesoscopic conductors at finite temperature was presented by Beenakker [1]. At a given energy, only the component of the emitted many-body state with 
one particle in detector region $\mathrm{A}$ and one in $\mathrm{B}$ has nonzero entanglement. Formally the entanglement of the emitted state, here called projected entanglement, is quantified in terms of the two-particle density matrix projected out from the many-body state. In an experiment, while this two-particle density matrix in principle can be projected out by local operations/measurements and classical communication between $\mathrm{A}$ and $\mathrm{B}$, it can not be directly accessed by standard measurements of currents and current correlators [8]. The reason for this is twofold:

First, at nonzero temperatures it is not only the biased source reservoirs which emit particles but also the grounded source reservoirs and the detectors do. As a consequence, there is a finite amplitude for emitted states with two-particles at A and/or at B. These unentangled states contribute to currents and current correlators, resulting in a detectable, effective state with suppressed entanglement. Second, the current and current correlators provide information on the energy integrated properties of the many-body state but not on the emitted state at each energy. This lack of energy-resolved information leads to a further suppression of the detectable entanglement. Clearly, these consequences of the thermally excited Fermi sea constitute generic problems in mesoscopic conductors.

As a remedy for these finite temperature read-out problems it was suggested to work with detectors at very low temperatures [1]. Recently, Hannes and Titov [9] investigated entanglement detection at finite temperatures via a Bell Inequality violation. In order to overcome the problem with detectors emitting particles they proposed to introduce energy filters, such as quantum dots at the drains. However, these schemes [1, 9] would lead to additional experimental complications in systems which already are experimentally very challenging. In this work we take a different route and investigate what information about the projected entanglement can actually be deduced from current and current correlation measurements. It is known [5] that such measurements allow for a complete, tomographic reconstruction of the reduced orbital two-particle density matrix. At the focus of our investigation will thus be the relation between the projected entanglement and the entanglement of the reduced density matrix, called the reduced entanglement.

To provide a physically compelling picture, we first investigate entanglement generation and detection in the 2PI. Thereafter a formal derivation for a general mesoscopic system is presented. We consider the 2PI shown in Fig. 1 with source reservoirs 1 and 2 biased at $\mathrm{eV}$ while 3 and 4 as well as the detector reservoirs are grounded [3]. All reservoirs are kept at the same temperature $T$. The reflectionless source beam-splitters $\mathrm{C}$ and $\mathrm{D}$ have transparencies $T_{C}=1-R_{C}$ and $T_{D}=1-R_{D}$ respectively. Note that all electrons impinging on the detectors are emitted by the source reservoirs; scattering between the detectors is prohibited in the 2PI-geometry.
In the 2PI-experiment, working with semi-transparent splitters $T_{C}=T_{D}=1 / 2$, a two-particle Aharonov-Bohm (AB) effect [3] was observed in the current cross correlations $S_{A+B+}$. For finite temperature and dephasing, theory [10] predicts

$$
S_{A+B+}=-e^{3} V /(4 h) H\left[1-\gamma \sin \phi_{t o t}\right]
$$

where $H=\operatorname{coth} x-1 / x$ with $x=e V / 2 k T, \gamma$ is a phenomenological decoherence parameter ranging from 1 for a fully coherent system to 0 for an incoherent one and $\phi_{\text {tot }}$, up to a constant, is the AB-phase. The applied bias $7.8 \mu \mathrm{V}$ and the estimated temperature $10 \mathrm{mK}$ in the experiment yield $H=0.78$. A direct comparison to Eq. (2) then gives the oscillation amplitude $H \gamma=0.25$, i.e. $\gamma=0.32$, a substantial dephasing. To determine the twoparticle entanglement of the emitted state we first calculate the projected (unnormalized) density matrix $\rho_{p}(E)$ at energy $E$. Using the formal similarity of the $2 \mathrm{PI}$ and the reflection-less, non spin-mixing splitter studied in [1], Eqs. (B9) - (B13), we get

$$
\rho_{p}(E)=(1-f)^{2} f_{V}^{2}\left[\chi \rho_{p}^{\text {diag }}+(1-\chi)^{2} \rho^{\text {int }}\right]
$$

where $\chi=e^{-2 x}$ and $f=1 /\left(1+e^{E / k T}\right)$ and $f_{V}=$ $1 /\left(1+\chi e^{E / k T}\right)$ the Fermi distribution functions of the grounded and biased source reservoirs respectively. We introduce a diagonal density matrix $\rho_{p}^{\text {diag }}=\chi \hat{1} \otimes \hat{1}+(1-$ $\chi)\left[\rho_{A} \otimes \hat{1}+\hat{1} \otimes \rho_{B}\right]$, where $\rho_{A}=R_{C}|+\rangle\left\langle+\left|+R_{D}\right|-\right\rangle\langle-|$ and $\rho_{B}=T_{C}|+\rangle\left\langle+\left|+T_{D}\right|-\right\rangle\langle-|$, and a density ma$\operatorname{trix} \rho^{\text {int }}=R_{C} T_{D}|+-\rangle\left\langle-+\left|+R_{D} T_{C}\right|-+\right\rangle\langle+-|-$ $\gamma \sqrt{T_{C} R_{C} T_{D} R_{D}}\left[e^{i \phi}|-+\rangle\left\langle-+\left|+e^{-i \phi}\right|+-\right\rangle\langle+-|\right]$ resulting from the two-particle interference, with $\phi$ a scattering phase. Here $\otimes$ is a direct product between single particle density matrices at $\mathrm{A}$ and $\mathrm{B}$ and $|-+\rangle \equiv|-\rangle_{A i}|+\rangle_{B i}$ with $\langle+-|=(|-+\rangle)^{\dagger}$ etc. The orbital states $|+\rangle_{A i / B i}$ $\left(|-\rangle_{A i / B i}\right)$ denote the upper (lower) incoming leads towards detector regions A/B (see Fig. 31). In agreement with Eq. (2), decoherence $\gamma<1$, is introduced as a suppression of the two-particle interference $\left|\Psi^{i n t}\right\rangle\left\langle\Psi^{i n t}\right| \rightarrow$ $\rho^{\text {int }}$, where $\left|\Psi^{i n t}\right\rangle=\sqrt{R_{C} T_{D}}|+-\rangle-e^{i \phi} \sqrt{T_{C} R_{D}}|-+\rangle$.

Following [1] we then write $\rho_{p}(E)=w_{p}(E) \sigma_{p}$ where $w_{p}(E)=\operatorname{tr}\left[\rho_{p}(E)\right]=(1-f)^{2} f_{V}^{2}\left[\left(R_{C} T_{D}+T_{C} R_{D}\right)(1-\right.$ $\left.\chi)^{2}+4 \chi\right]$ and $\sigma_{p}$ the normalized, energy independent density matrix of the emitted two-particle state. The entanglement production $C_{p}(E) \equiv w_{p}(E) C\left(\sigma_{p}\right)$ is conveniently quantified in terms of the concurrence $C$ [11], ranging from 0 for a separable state to 1 for a maximally entangled state. The total entanglement production in a time $\tau, C_{p}=(\tau / h) \int d E C_{p}(E)$, is then $(\mathcal{N}=\tau e V / h)$

$$
C_{p}=(\mathcal{N} H / 2) \max \left\{4 \gamma \sqrt{T_{C} R_{C} T_{D} R_{D}}-\sinh ^{-2} x, 0\right\} .
$$

As shown in Fig. 2] $C_{p}$ decreases monotonically as a function of $T$, reaching zero at a critical temperature $T_{c}^{p}$.

Inserting the parameter values from the experiment, we find that $C_{p} \approx 0.1 \mathcal{N}$ and $C\left(\sigma_{p}\right) \approx 0.3$, i.e. the state 

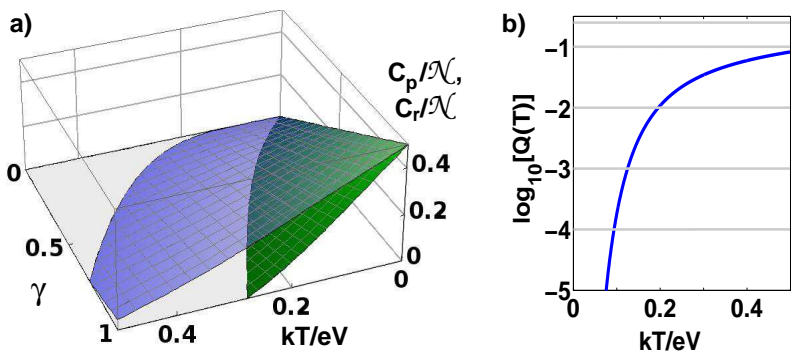

FIG. 2: (color online) a) Entanglement production $C_{p} / \mathcal{N}$ (blue, transparent) and $C_{r} / \mathcal{N}$ (green, opaque) as functions of temperature $k T / e V$ and coherence $\gamma$ for the semi-transparent 2PI. b) Parameter $Q$ as a function of $k T / e V$ (blue line). Values $0.25,10^{-1}, 10^{-2}, 10^{-3}, 10^{-4}$ shown (grey lines).

emitted by the 2PI is clearly entangled. However, this is a rather indirect deduction of the two-electron entanglement, an unambiguous demonstration of entanglement would be desirable. For this we turn to the reduced density matrix $\rho_{r}$, providing full information about the detectable two-particle correlations.

We first express $\rho_{r}$ in terms of currents $I_{A \alpha}, I_{B \beta}$ and low-frequency current cross correlators [8] $S_{A \alpha B \beta}$, with $\alpha, \beta= \pm$. Extending [5] to nonzero temperatures we find

$$
\frac{I_{A \alpha} I_{B \beta}}{\left(V e^{2} / h\right)^{2}}+\frac{S_{A \alpha B \beta}}{2 V e^{3} / h}=\operatorname{tr}\left\{\left[I_{A \alpha}^{O} \otimes I_{B \beta}^{O}\right] \rho_{r}\right\} .
$$

The orbital current operators in the local basis $\{| \pm\rangle\}$, including the rotations at the detector splitters, are $I_{A \alpha}^{O}=\left(\hat{1}+\alpha S_{A} \sigma_{z} S_{A}^{\dagger}\right) / 2$ and $I_{B \beta}^{O}=\left(\hat{1}+\beta S_{B} \sigma_{z} S_{B}^{\dagger}\right) / 2$, with $\sigma_{z}$ the Pauli matrix and $S_{A}\left(S_{B}\right)$ the scattering matrix of the splitter at A (B). The results of Ref. 10] yield

$$
\rho_{r}=(1-H)\left[\rho_{A} \otimes \rho_{B}\right]+H \rho^{i n t} .
$$

Writing $\rho_{r}=w_{r} \sigma_{r}$ with $w_{r}=\operatorname{tr}\left[\rho_{r}\right]=\left[R_{C} T_{C}+\right.$ $\left.R_{D} T_{D}\right](1-H)+R_{C} T_{D}+R_{D} T_{C}$ and $\sigma_{r}$ the normalized density matrix we then define the total entanglement production during a time $\tau$ as $C_{r} \equiv \mathcal{N} w_{r} C\left(\sigma_{r}\right)$. It is

$$
C_{r}=2 \mathcal{N} \max \left\{\sqrt{T_{C} R_{C} T_{D} R_{D}}[H(1+\gamma)-1], 0\right\} .
$$

As $C_{p}, C_{r}$ decreases monotonically with increasing $T$, reaching zero at $T=T_{c}^{r}$, as shown in Fig. 2,

Comparing Eqs. (44) and (17) we find that $C_{p} \geq C_{r}$ for $Q(T)=H /\left[4(1-H) \sinh ^{2} x\right] \leq \sqrt{T_{C} R_{C} T_{D} R_{D}}$, independent on $\gamma$ (see Fig. 2). Consequently, for splitters away from the strongly asymmetrical, tunneling limit, the reduced entanglement constitutes a lower bound for the projected entanglement. In the tunneling limit, however, the reduced entanglement is larger than the projected one. This is the case since asymmetry has different effects on $C_{p}$ and $C_{r}$; the critical temperature $T_{c}^{r}$, from Eq. (7), is independent on the splitter transparencies $T_{C}, T_{D}$ while $T_{c}^{p}$, from Eq. (4), is reduced by increasing asymmetry. For the parameters in the experiment,
$Q(T) \approx 4 \times 10^{-4} \ll \sqrt{R_{C} T_{C} R_{D} T_{D}} \approx 0.25$, showing the validity of the bound. However, $C_{r} \approx 0.01 \mathcal{N}$ and based on the measurement [4] no conclusive statement can be made about $C_{r}$ and hence not about $C_{p}$.

A more detailed understanding of this finite temperature readout problem can be obtained by comparing the properties of $\sigma_{p}$ and $\sigma_{r}$. For perfect coherence $\gamma=1$ and identical beam splitters $T_{C}=T_{D}=\mathcal{T}=1-\mathcal{R}$ one can (up to a local phase rotation) write $\sigma_{p / r}=$ $\frac{1}{4} \xi_{p / r} \hat{1} \otimes \hat{1}+\left(1-\xi_{p / r}\right)\left|\Psi_{s}\right\rangle\left\langle\Psi_{s}\right|$, a Werner state [12], with singlet weight [1] $1-\xi_{p}=2 \mathcal{R} \mathcal{T} \sinh ^{2} x /\left[1+2 \mathcal{R} \mathcal{T} \sinh ^{2} x\right]$ and $1-\xi_{r}=H /(2-H)$. Increasing $k T / e V=2 / x$ from zero, $\xi_{p} \approx 2 e^{-2 x} /(\mathcal{R} \mathcal{T})$ becomes exponentially small while $\xi_{r} \approx 2 / x$ increases linearly. These qualitatively different behaviors, clearly illustrated in Fig. 1, are a striking signature of how a small $k T / \mathrm{eV}$, having negligible effect on $C\left(\sigma_{p}\right)$, leads to a large suppression of $C\left(\sigma_{r}\right)$.

From Eqs. (4) and (7) follows also a counter-intuitive result: finite amplitude of the AB-oscillations is no guarantee for finite two-particle entanglement. This is apparent for $\sigma_{r}$ in the limit $\gamma=1$ and $T_{C}=$ $T_{D}$, since a separable Werner state, $\xi_{r}>2 / 3$, can be decomposed [13] into a sum of product states as $\sigma_{r}=\frac{1}{4} \sum_{n=1}^{4}\left|\phi_{n}^{A}\right\rangle\left\langle\phi_{n}^{A}|\otimes| \phi_{n}^{B}\right\rangle\left\langle\phi_{n}^{B}\right|$ with $\left|\phi_{n}^{A / B}\right\rangle=$ $\cos \theta_{n}^{A / B}|+\rangle+e^{i \pi[1-2 n] / 4} \sin \theta_{n}^{A / B}|-\rangle$ and $\theta_{1}^{A / B}=\theta_{3}^{A / B}=$ $\operatorname{atan}\left[y^{A / B}\right], \theta_{2}^{A / B}=\theta_{4}^{A / B}=-\operatorname{acot}\left[y^{A / B}\right]$ and $y^{A / B}=$ $\left(\sqrt{2-\xi_{r}}+\sqrt{3 \xi_{r}-2}\right) /\left(\sqrt{\xi_{r}} \pm \sqrt{4-3 \xi_{r}}\right)$ with $+(-)$ for A(B). This classically correlated state gives, via Eq. (5), AB-oscillations with amplitude $2\left(1-\xi_{r}\right) /\left(2-\xi_{r}\right)=H$.

Moreover, the effect of decoherence, suppressing the two-particle interference, is similar for the projected and the reduced entanglement. In particular, for $T=0$; $C_{p}=C_{r}=2 \mathcal{N} \gamma \sqrt{R_{C} T_{C} R_{D} T_{D}}$, finite for arbitrary strong dephasing [3]. This is a consequence of the 2PI-geometry, prohibiting scattering between upper $(+)$ and lower $(-)$ leads, i.e. pseudo-spin flips [3, 6, 14]. Importantly, given the controllability of phase gates and beam splitters [15], demonstrated in the electronic Mach-Zehnder and 2PI experiments [4, 16], all the technics necessary for an entanglement test via reconstruction of $\rho_{r}$ are at hand.

Another widely discussed [3, 6, 7, 17] approach to detect the entanglement is to use a Bell inequality. Violation of a CHSH-Bell inequality [18] formulated in terms of currents and low-frequency current correlations demonstrates finite entanglement of $\rho_{r}$. An optimal CHSH-Bell test demands the same number of measurement and level of experimental complexity as a tomographic reconstruction of $\rho_{r}$. From $\sigma_{p}$ and $\sigma_{r}$, we can using [19] calculate the corresponding maximal Bell parameters $\Omega_{B p}^{\max }$ and $\Omega_{B r}^{\max }$, yielding for identical source splitters $T_{C}=T_{D}=\mathcal{T}$

$$
\Omega_{B p / r}^{\max }=2 \sqrt{1+\gamma^{2}}\left(1-\xi_{p / r}\right)
$$

The CSHS-Bell inequalities are $\Omega_{B p / r} \leq 2$, with the limits $\Omega_{B p / r}^{\max }=2$ for $\mathcal{T}=1 / 2$ plotted in Fig. 1. It is clear that for the values $k T / e V$ and $\gamma$ of the 2PI-experiment, 
while $\Omega_{B p} \leq 2$ in principle can be violated, a detection of entanglement by violating $\Omega_{B r} \leq 2$ is not possible.

Extending the above analysis to a general mesoscopic system, it can be shown 20] that away from the strongly asymmetric limit (see below), $C_{r}>0$ guarantees $C_{p}>0$, or equivalently $T_{c}^{p} \geq T_{c}^{r}$, i.e. for a general conductor, nonzero entanglement of $\rho_{r}$ demonstrates that the emitted many-body state is entangled. This motivates a detailed investigation of the $\rho_{r}$ and $C_{r}$ in a general system.

We consider a conductor, shown in Fig. 3, characterized by a scattering matrix $S$ and connected via single mode leads to $M \geq 2$ reservoirs biased at $\mathrm{eV}$ and $N-M \geq 0$ grounded. The conductor is also connected to detector reservoirs $A \alpha$ and $B \beta$ via reflectionless splitters with controllable phase gates. The splitter-phase gate structures perform local rotations, characterized by $S_{A}$ and $S_{B}$, of the orbital states. All reservoirs are kept at temperature $T$. We assume linear response in applied bias and $S$ independent on energy $E$ in the interval $-k T \lesssim E \lesssim e V+k T$ of interest. The scattering is moreover assumed to be spin independent and we hence drop spin notation, presenting results for a single spin species.

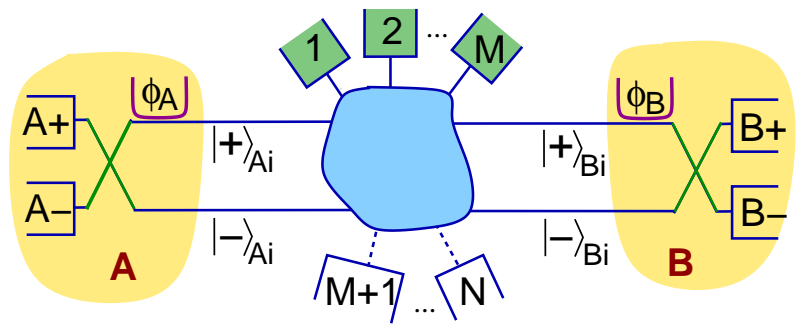

FIG. 3: (color online) Sketch of the general system. A mesoscopic scatterer is connected to $M$ biased and $N-M$ grounded source reservoirs and, via phase gates $\phi_{A / B}$ and beam splitters, to grounded detector reservoirs $A \pm, B \pm$. The orbital basis states $| \pm\rangle_{A i / B i}$ are displayed. For details see text.

In order to treat general conductors we need to modify Eq. (5) as $S_{A \alpha B \beta} \rightarrow S_{A \alpha B \beta}^{t r}$ where $S_{A \alpha B \beta}^{t r} \equiv$ $S_{A \alpha B \beta}(e V, k T)-S_{A \alpha B \beta}(0, k T)$, the non-equilibrium excess transport part of the correlator [8]. Working in the orbital basis $\{|++\rangle,|+-\rangle,|-+\rangle,|--\rangle\}$, we get from scattering theory [8] the reduced density matrix

$$
\rho_{r}=t_{A} t_{A}^{\dagger} \otimes t_{B} t_{B}^{\dagger}-H\left(t_{A} t_{B}^{\dagger} \otimes t_{B} t_{A}^{\dagger}\right) P
$$

where $P$ is a $4 \times 4$ permutation matrix with nonzero elements $P_{i j}=1$ for $i j=\{11,23,32,44\}$. The $2 \times M$ transmission matrix $t_{A}\left(t_{B}\right)$ is the matrix amplitude for scattering from the $M$ biased reservoirs to the two leads going out from the source towards region A (B).

To obtain a compact expression for $C_{r}$ we make the singular value decompositions $t_{A}=U_{A} \mathcal{T}_{A} V_{A}$ and $t_{B}=U_{B} \mathcal{T}_{B} V_{B}$, where the $2 \times M$ matrix $\mathcal{T}_{A}=\left[\tau_{A}, 0\right]$ with $\tau_{A}=\operatorname{diag}\left(\sqrt{T_{A+}}, \sqrt{T_{A-}}\right)$ and similar for $\mathcal{T}_{B}$, and
$U_{A}, V_{A}, U_{B}, V_{B}$ unitary. Inserting this decomposition into $\rho_{r}$ in Eq. (9) we arrive after some algebra at

$$
\begin{aligned}
C_{r} & =2 \mathcal{N} \sqrt{T_{A+} T_{A-} T_{B+} T_{B-}} \max \{F, 0\} \\
F & =H \sqrt{\zeta_{1} \zeta_{2}}-\sqrt{\left(1-H \zeta_{1}\right)\left(1-H \zeta_{2}\right)}
\end{aligned}
$$

where $\zeta_{1}, \zeta_{2} \in[0,1]$ are the eigenvalues of $Z Z^{\dagger}$, with $Z$ a $2 \times 2$ matrix with elements $Z_{i j}=\left(V_{A}^{\dagger} V_{B}\right)_{i j}$ for $i, j=1,2$.

It can be shown 20] that for $\chi /[(1-\chi)(1-H)]<$ $\left(T_{A+}^{-1}+T_{A-}^{-1}+T_{B+}^{-1}+T_{B-}^{-1}\right)^{-1}, C_{r}>0$ guarantees $C_{p}>$ 0 . This condition is expressed in terms of $T_{A \pm}, T_{B \pm}$, eigenvalues of the reduced single particle density matrices accessible by a reconstruction using average currents [5].

In conclusion, we have presented a theory for entanglement generation and detection in mesoscopic conductors at finite temperatures. It is found that under very general conditions finite reduced, detectable entanglement constitutes a witness for nonzero emitted, projected entanglement. The theory applied to the two-particle interferometer [3] investigated experimentally by Neder et al [4] shows that while the emitted state is clearly entangled, the detectable entanglement is close to zero. Our results provide motivation for further experimental investigations of entanglement in the 2PI.

We acknowledge discussions with E.V. Sukhorukov. The work was supported by the Swedish VR, the Israeli SF, the MINERVA foundation, the German Israeli Foundation (GIF) and Project Cooperation (DIP), the USIsrael Binational SF, the Swiss NSF and MaNEP.

[1] C.W.J. Beenakker, in Proc. Int. School Phys. E. Fermi, Vol. 162, Quantum Computers, Algorithms and Chaos, eds. G. Casati et al (IOS Press, Amsterdam, 2006).

[2] P. Recher, D. Saraga, D. Loss, in Fundamental Problems of Mesoscopic Physics Interaction and Decoherence, eds. I.V. Lerner et al., NATO Science Ser. II, Vol. 154 (Kluwer, Dordrecht, 2004)

[3] P. Samuelsson, E.V. Sukhorukov, M. Büttiker, Phys. Rev. Lett. 92, 026805 (2004).

[4] I. Neder et al, Nature 448, 333 (2007).

[5] P. Samuelsson, M. Büttiker, Phys. Rev. B 73, 041305 (2006).

[6] P. Samuelsson, E.V. Sukhorukov, M. Büttiker, Phys. Rev. Lett. 91, 157002 (2003).

[7] C.W.J. Beenakker et al., Phys. Rev. Lett. 91, 147901 (2003).

[8] M. Büttiker, Phys. Rev. B 4612485 (1992); Ya. Blanter, and M. Büttiker, Phys. Rep. 336, 1 (2000).

[9] W.-R. Hannes, M. Titov, Phys. Rev. B 77, 115323 (2008).

[10] V.S.-W. Chung, P. Samuelsson, M. Büttiker, Phys. Rev. B 72, 125320 (2005).

[11] W. K. Wootters, Phys. Rev. Lett. 80, 2245 (1998).

[12] R. F. Werner, Phys. Rev. A 40, 4277 (1989).

[13] J. Samsonowicz, M. Kuz, and M. Lewenstein Phys. Rev. A 76, 022314 (2007). 
[14] J.L. van Velsen, M. Kindermann, and C.W.J. Beenakker, Turk. J. Phys. 27, 323 (2003); P. Samuelsson, E.V. Sukhorukov, M. Büttiker, ibid, 27481 (2003)

[15] M. Henny et al., Science 284, 296 (1999); Oliver et al., ibid. 284, 299 (1999); S. Oberholzer et al., Physica E 6, 314 (2000).

[16] Y. Ji, et al., Nature 422, 415 (2003); Neder et al, Phys. Rev. Lett. 96016804 (2006); L. V. Litvin et al., Phys. Rev. B 75, 033315 (2007); P. Roulleau et al., Phys. Rev. Lett. 100, 126802 (2008); E. Bieri, PhD-thesis, University of Basel (2007).
[17] N.M. Chtchelkatchev et al., Phys. Rev. B 66, 161320 (2002); L. Faoro, F. Taddei, and R. Fazio, Phys. Rev. B 69, 125326 (2004); A. V. Lebedev, G. B. Lesovik, and G. Blatter, Phys. Rev. B 71, 045306 (2005).

[18] J.F. Clauser et al. Phys. Rev. Lett. 23, 880 (1969).

[19] R. Horodecki, P. Horodecki, and M. Horodecki, Phys. Lett. A 200, 340 (1995); F. Verstraete, and M.M. Wolf, Phys. Rev. Lett. 89, 170401 (2002).

[20] P. Samuelsson, I. Neder, and M. Büttiker, in preparation. 